\title{
Predictability of Extratropical Upper-Tropospheric Circulation in the Southern Hemisphere by Its Main Modes of Variability $\mathscr{O}$
}

\author{
MARISOL OSMAN AND CAROLINA S. VERA \\ Universidad de Buenos Aires, Facultad de Ciencias Exactas y Naturales, Departamento de Ciencias de la Atmósfera y los \\ Océanos, and Universidad de Buenos Aires, Consejo Nacional de Investigaciones Científicas y Técnicas, CNRS, \\ Centro de Investigaciones del Mar y la Atmósfera, and Instituto Franco-Argentino sobre Estudios de Clima \\ y sus Impactos (UMI IFAECI), Buenos Aires, Argentina
}

(Manuscript received 15 February 2019, in final form 8 November 2019)

\begin{abstract}
The predictability and forecast skill of the models participating in the Climate Historical Forecast Project (CHFP) database is assessed through evaluating the representation of the upper-tropospheric extratropical circulation in the Southern Hemisphere (SH) in winter and summer and its main modes of variability. In summer, the predictability of 200-hPa geopotential height anomalies mainly comes from the ability of the multimodel ensemble mean (MMEM) to forecast the first three modes of interannual variability with high fidelity. The MMEM can reproduce not only the spatial patterns of these modes but also their temporal evolution. On the other hand, in JJA only the second and fourth modes of variability are predictable by the MMEM. These seasonal differences in the performance of the MMEM seem to be related to the role that the sea surface temperature (SST) anomalies have in influencing the variability of each mode. Accordingly, modes that are strongly linked to tropical SST anomalies are better forecast by the MMEM and show less spread among models. The analysis of both 2-m temperature and precipitation anomalies in the SH associated with the predictable modes reveals that DJF predictable modes are accompanied by significant temperature anomalies. In particular, temperatures at polar (tropical) latitudes are significantly correlated with the first (second) mode. Furthermore, these links obtained with observations are also well forecast by the MMEM and can help to improve seasonal forecast of climate anomalies in those regions with low skill.
\end{abstract}

\section{Introduction}

The implementation of climate services at local scales requires the development of skillful regional prediction tools. In the Northern Hemisphere (NH), several works have addressed the levels of predictability attained by climate models on seasonal time scales using multimodel ensembles (Jha et al. 2019). However, the levels of predictability and prediction skill on seasonal time scales in the Southern Hemisphere ( $\mathrm{SH})$, as well as their associated sources, has not been fully assessed yet. Osman et al. (2016) performed a first assessment of the tropospheric circulation in the SH considering models participating in the World

Supplemental information related to this paper is available at the Journals Online website: https://doi.org/10.1175/JCLI-D-190122.s1.

Corresponding author: Marisol Osman, osman@cima.fcen. uba.ar
Climate Research Programme (WCRP) Climate Historical Forecast Project (CHFP). They show that predictability (signal-to-variance ratio) and prediction skill (described by anomaly correlation between predictions and observations) are higher in the tropics than in the extratropics and are also higher in summer than in winter. Nevertheless, modest values of predictability and skill are found at specific extratropical regions, such as near the Antarctic Peninsula, over the Bellingshausen-Amundsen Seas, and in the central South Pacific Ocean. Previous studies showed that climate variability in these extratropical regions is largely influenced by that of the leading patterns of circulation variability in the SH (e.g., Mo 2000). The latter then opens the questions about how much of the extratropical predictability is explained by that associated with the main modes of circulation variability.

The main modes of variability of tropospheric circulation in the $\mathrm{SH}$ have been described in the literature mainly by using geopotential height anomalies, and the activity of some of them has been at least partially 
related to the sea surface temperature (SST) variability at the tropics. The first leading mode, known in the literature as the Antarctic Oscillation or southern annular mode (SAM), exhibits a highly zonally symmetric structure that describes an anomaly reversal between polar and middle latitudes (Kidson 1999; Mo 2000). This mode, active all year around, is associated with the intensification or weakening of the westerlies, and it has a barotropic equivalent structure. The next two modes describe Rossby wave-like patterns extending from the tropical Pacific and Australian sector to southern South America. They are generally referred to as PacificSouth America (PSA) patterns (e.g., Ghil and Mo 1991; Mo 2000). The second leading mode, usually called PSA1 (e.g., Mo 2000), is associated with the SST variability mainly at the central-eastern Pacific, related to El Niño-Southern Oscillation (ENSO). On the other hand, the third leading mode, usually called PSA2 (Mo 2000), is linked to SST anomalies in the tropical Indian Ocean with dominant periodicity at around $2-3$ years. PSA patterns are associated to enhanced convection in the tropics as the major atmospheric response to ENSO and warm SST over the Maritime Continent in the SH. The two PSA patterns are active all year around but with some seasonal variations in their amplitude. Both influence regional temperature and precipitation anomalies in Australia, New Zealand, Antarctica, and southern South America (Berman et al. 2012; Fogt et al. 2011; Silvestri and Vera 2009; Hendon et al. 2007).

The traditional way to determine predictability encompasses the computation of the ratio between the variance of signal (associated to external forcings) against the total variance (forced variance plus internal variance). However, as results based on this type of approach seem to be model dependent (e.g., Wu and Kirtman 2006), alternative strategies have been suggested to estimate predictability of seasonal scales. Wang et al. (2007) introduce the "predictable mode analysis," which estimates predictability by the fractional variance accounted by the predictable leading modes of variability identified in both observations and hindcast multimodel ensemble mean (MMEM) forecasts. Lee et al. (2011) applied it to assess the predictability of summer upper-level circulation in the $\mathrm{NH}$, and confirm that it comes mainly from the skill in predicting the first two leading patterns. Moreover, the predictability assessment of the Asian winter temperature variability made by Lee et al. (2013) shows that it is mainly explained by the predictability of the first four associated leading modes.

Therefore, in this work the predictable mode analysis is applied to assess the contribution of the three leading patterns of tropospheric extratropical circulation variability in the $\mathrm{SH}$ to the overall predictability on seasonal time scales. The study concentrates on austral summer and winter, and it uses the WCRP-CHFP (Tompkins et al. 2017) database. This is the first time in which this methodology is applied in the $\mathrm{SH}$, in contrast to the $\mathrm{NH}$, where it has been applied before. In addition, the focus is on assessing the agreement between and within CHFP models in reproducing those predictable modes, which represents an advance with respect to previous works (e.g., Grainger et al. 2011, 2013) that focused on the ensemble mean of the models.

The study is organized as follows: section 2 describes the model and observational data used in this work along with the details of the methodology implemented. Section 3 describes the main modes of variability resulting from observations and model predictions, their sources of predictability, and the assessment of both the skill and potential predictability achieved by those modes. Finally, section 4 discusses the climate anomalies associated with the predictable modes.

\section{Data and methodologies}

\section{a. Model data}

Seasonal hindcasts from nine institutions participating in the WCRP CHFP-SHFP Project (available at http:// chfps.cima.fcen.uba.ar) are considered. Model details, such as horizontal and vertical resolution and ensemble size, are described in Table 1. Monthly means of geopotential height at $200 \mathrm{hPa}, 2-\mathrm{m}$ air temperature, and precipitation rate over the Southern Hemisphere for December-February (DJF) and June-August (JJA), from initial conditions observed in November and May respectively (lead 1 month), are considered for the period 1982-2005. The multimodel ensemble mean prediction was made by simply averaging the ensemble means of the 15 models. In addition, SST predictions from the same models are also considered.

\section{b. Observed data}

NCEP-NCAR reanalysis data (Kalnay et al. 1996) were considered as observations for $200-\mathrm{hPa}$ geopotential heights and 2-m air temperature. Observed precipitation data were obtained from the Climate Prediction Center (CPC) merged analysis of precipitation (CMAP) dataset (Xie and Arkin 1997), while observed SST data from the improved Extended Reconstructed Sea Surface Temperature version 2 (ERSST V2) dataset (Smith and Reynolds 2004).

\section{c. Methodology}

We used the "predictable modes analysis" approach developed by Wang et al. (2007) to assess the predictability of upper-level tropospheric circulation in DJF 
TABLE 1. Description of the models used in this study. (Expansions of acronyms are available online at http://www.ametsoc.org/ PubsAcronymList.)

\begin{tabular}{|c|c|c|c|c|}
\hline Institution/acronym & Model & Resolution & Ensembles & References \\
\hline \multirow[t]{2}{*}{ Météo France } & ARPEGE 4.4-OPA & T63L91 (0.01 hPa) & 11 & Guérémy et al. (2005) \\
\hline & & T63L31 $(10 \mathrm{hPa})$ & & \\
\hline \multirow[t]{2}{*}{$\mathrm{CCCma}$} & CMAM & T63L71 (100 km) & 10 & Scinocca et al. (2008) \\
\hline & & T63L41 (31 km) & & \\
\hline \multirow[t]{2}{*}{ CCCma } & CanCM3-CGCM3 & T63L31 (1 hPa) & 10 & Scinocca et al. (2008) \\
\hline & CanCM4-CGCM4 & T63L35 (1 hPa) & & Arora et al. (2011) \\
\hline NCEP & CFSV1 (GFS-MOM3) & T62L64 (0.2 hPa) & 7 & Saha et al. (2006) \\
\hline JMA/MRI & GSM-MOVE & TL95L40 $(0.4 \mathrm{hPa})$ & 10 & Yukimoto et al. (2012) \\
\hline \multirow[t]{2}{*}{ ECMWF } & System 3 (IFS-HOPE) & T159L62 (5 hPa) & 9 & Stockdale et al. (2011) \\
\hline & System 4 (IFS-NEMO) & TL255L91 $(0.01 \mathrm{hPa} 74 \mathrm{~km})$ & 15 & Molteni et al. (2011) \\
\hline MPI & ECHAM6-MPIOM & T63L47 $(0.01 \mathrm{hPa})$ & 9 & $\begin{array}{l}\text { Stevens et al. (2013); } \\
\quad \text { Marsland et al. (2003) }\end{array}$ \\
\hline AORI/NIES/JAMSTEC & MIROC5 & T85L40 (3 hPa) & 8 & Watanabe et al. (2010) \\
\hline \multirow[t]{3}{*}{ BoM } & POAMA P24a & T44L17 & 10 & Colman et al. (2005); \\
\hline & POAMA P24b & & & Schiller et al. (2008) \\
\hline & POAMA P24c & & & \\
\hline
\end{tabular}

and JJA. It is based on the identification of the main modes of interannual variability for both observations and MMEM predictions. Predictability is then estimated as the percentage of variance explained by the predictable modes.

The predictable modes were first identified by an empirical orthogonal function (EOF) analysis (based on the correlation matrix) of the $200-\mathrm{hPa}$ geopotential height anomalies over the extratropical $\mathrm{SH}\left(20^{\circ}-90^{\circ} \mathrm{S}, 0^{\circ}-360^{\circ} \mathrm{E}\right)$. The analysis was performed separately to observations and MMEM predictions. Then, the first components were rotated using the varimax technique to maximize the local variance within the domain. The number of rotated modes was chosen following O'Lenic and Livezey (1988).

We measured the forecast performance through the computation of the temporal correlation coefficient (TCC) score for temporal variations of the times series or principal components (PCs) and the pattern correlation coefficient (PCC) score for spatial variations in the eigenvectors. As in Lee et al. (2011), we defined a skill score that represents the coupled models' capability in predicting EOF modes, combining for each mode the PCC score for eigenvectors and TCC score for the PC time series, as follows:

$$
\text { skillscore }=\sqrt{\mathrm{PCC} \times \mathrm{TCC}} .
$$

The skill score ranges from 0 (no skill at all) to 1 (perfect forecast). We reordered the EOF modes of the MMEM prediction according to the PCC skill as the order of the predicted EOF mode is not necessarily the same as its observed counterpart. The arrangement process encompasses the computation of the PCC skill for the observed leading mode against all predicted modes. Then the predicted mode exhibiting the highest PCC skill with the observed leading mode was selected as the predicted leading mode. Repeating the above process with the remaining modes, the rest of the predicted modes were similarly determined. In the case of $200-\mathrm{hPa}$ geopotential height, the first predicted mode matches with the observed mode in both seasons considered, while the other modes had to be reordered. Those exhibiting significant PCC as well as significant TCC were considered as the "predictable modes."

In addition, we also performed an analysis of the predictable modes by applying rotated EOF (REOF) analysis to 1) the ensemble constructed with the anomaly of each model ensemble mean with respect to the MMEM (MMEa) and 2) the ensemble constructed with the anomalies of all ensemble members from all models with respect to the ensemble mean of each model (SMMEa). In each analysis, an eigenvector was obtained for each mode, which represents the pattern that maximizes the variance explained by all the members of the ensemble considered. In addition, the analysis provides a PC for each ensemble member after projecting that member on the eigenvector. This analysis is similar to the analysis of variance (ANOVA) developed by Hodson and Sutton (2008). A detailed description of the methodology can be found in Hodson and Sutton (2008) and von Storch and Zwiers (2002). ANOVA proposes a decomposition of an individual data value $X_{t m k}$ (where $t, m$, and $k$ denote year, model, and ensemble member, respectively) as follows:

$$
X_{t m k}=\mu+\alpha_{t}+\beta_{m}+\gamma_{t m}+\varepsilon_{t m k}
$$

where $\mu$ represents the time-mean ensemble-mean model mean, $\alpha_{t}$ is the time-varying behavior that is common to all ensemble members from all models, $\beta_{m}$ is the bias of a 
a)

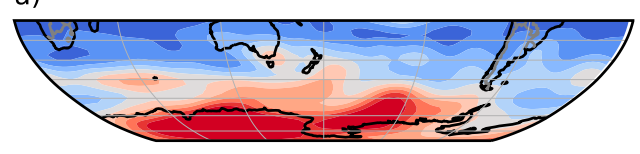

c)

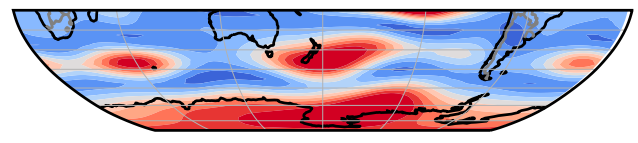

e)

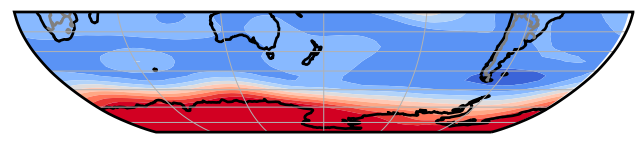

b)

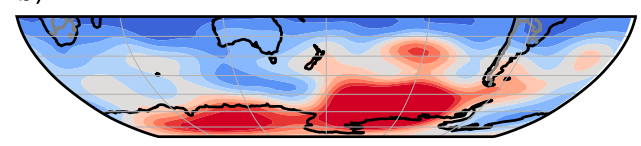

d)

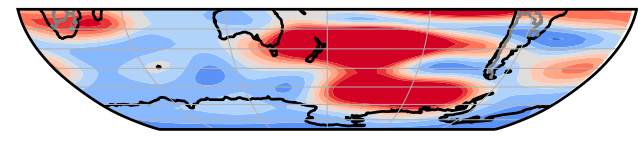

f)

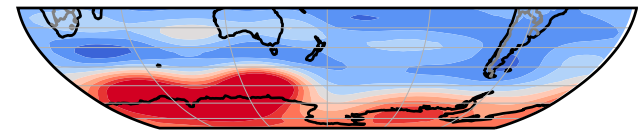

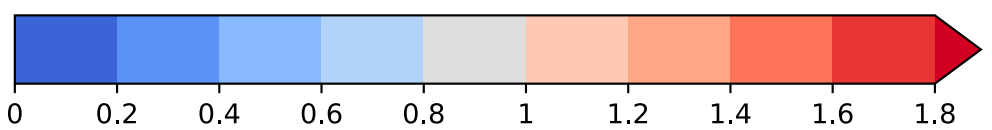

FIG. 1. Ratio between the 200 -hPa geopotential height variance at each grid point and the mean variance of the domain for (a) DJF and (b) JJA observations, (c) DJF and (d) JJA MMEM, and (e) DJF and (f) JJA MMEa.

given model, $\gamma_{t k}$ is the unbiased time-varying differences between the ensemble means of the models and captures the difference in the model's response to the same forcing, and $\varepsilon_{t m k}$ is the residual noise. In our study, the REOF analysis applied to the MMEM identifies the main modes of variability associated to $\alpha_{t}$, whereas the REOF analysis applied to the MMEa does it with the mean differences between the ensemble means of the models (in which the bias is included). Finally, the REOF analysis applied to the SMMEa provides the modes associated with $\varepsilon_{t m k}$. The objective of this approach is twofold: it increases the size of the sample considered while helping to analyze the patterns associated with intermodel and internal variability. In this sense, the proposed analysis represents an improvement in the decomposition of the variance from previous studies done for the SH (e.g., Grainger et al. 2011, 2013) in which the interaction between the ensemble members of models has not been assessed.

\section{Results}

a. DJF

\section{1) DESCRIPTION OF VARIABILITY AND IDENTIFICATION OF PREDICTABLE MODES}

In this section we describe the interannual variability of DJF SH upper-tropospheric circulation by both observations and the MMEM. Figure 1 shows the variance of DJF 200-hPa geopotential heights for observations as well as for predictions resulting from both MMEM and MMEa. To facilitate the comparison, given that observed variability is much higher than model variability, variances are presented as relative to the corresponding spatially averaged variance. In DJF, observed variability exhibits the largest amplitude at polar latitudes, over east Antarctica and the AmundsenBellingshausen and Ross Seas, where values double the spatial mean variability (Fig. 1a). High values are also discernible over the Indian Ocean and south of New Zealand. Near the tropics, variability is less than $50 \%$ of the spatial mean variability. The MMEM also presents largest values of variability at polar latitudes although only the maximum over the Amundsen-Bellingshausen Seas is captured (Fig. 1c). At midlatitudes, the MMEM exhibits three distinctive regions of high variance that are not discernible in observations. At the tropics, observations show minimum values over the Pacific while the MMEM presents a local maximum there. The ratio of variance of the MMEa is zonally uniform, showing a marked contrast between the polar to middle latitudes and the tropics (Fig. 1e). Nevertheless, the maximum observed at the poles in previous figures is also discernible but shifted to eastern Antarctica, doubling mean variances, while most of the domain presents around $50 \%$ of the spatial mean variability. 
The ability of the MMEM to describe the main modes of variability of the 200-hPa geopotential heights in DJF was also assessed. Figure 2 shows the scatterplot linking, for each mode, the percentage of observed variance and the corresponding MMEM skill score. The first mode is the only one that has an explained variance well separated from the rest of the modes. However, given that skill scores for the three leading modes are significant, we consider them also predictable. These modes explain about $51 \%$ of the observed variance and $87 \%$ of the MMEM variance.

Figure 3 shows the spatial distribution of the three leading modes identified after applying the REOF analysis to observations (first column), the MMEM (second column), the MMEa (third column), and the SMMEa (fourth column), respectively. In addition, Fig. 4 shows the corresponding PCs while Table 2 summarizes the PCC as well as TCC computed between the observed modes and those resulting from the three ensemble types. The first mode explains approximately $31 \%$ of the observed total variance. It is characterized by a monopole of negative geopotential height anomalies in the pole and four centers of positive anomalies at midlatitudes (Fig. 3a). The mode is associated with the intensification and lessening of the westerlies and is usually known as the SAM, as discussed in the introduction. The second mode explains about $11 \%$ of the observed variance and combines a zonally stretched pattern of positive anomalies at tropics and a wave train extending toward the extratropics (Fig. 3e) with positives anomalies over southern South America and the southwestern Pacific Ocean and negative anomalies from the southwestern Indian Ocean and southern Australia to the central South Pacific Ocean. The associated PC (Fig. 4b) maximizes in ENSO years such as 1982/83, 1987/ 88 , and $1997 / 98$. The mode is usually called PSA1 in the literature, as described in the introduction. The third mode, which accounts for about $8 \%$ of the observed variance, presents a wavelike pattern in the entire domain. Positive anomalies are found in the central tropical Pacific Ocean, the Amundsen-Bellingshausen and Ross Seas, the southwestern Atlantic Ocean, and southern Africa while negative anomalies are found over the central South Pacific Ocean, southeastern Atlantic Ocean, and southeastern Indian Ocean (Fig. 3i). It is usually called PSA2 in the literature.

We now analyze the modes obtained in the REOF analysis applied to the MMEM, which captures the main variability common to all ensemble members. The MMEM is able to reproduce, spatially and temporally, the main features of the three main modes of variability. The percentage of the total variance explained by each mode is $47 \%, 18 \%$, and $23 \%$, respectively. The PCC

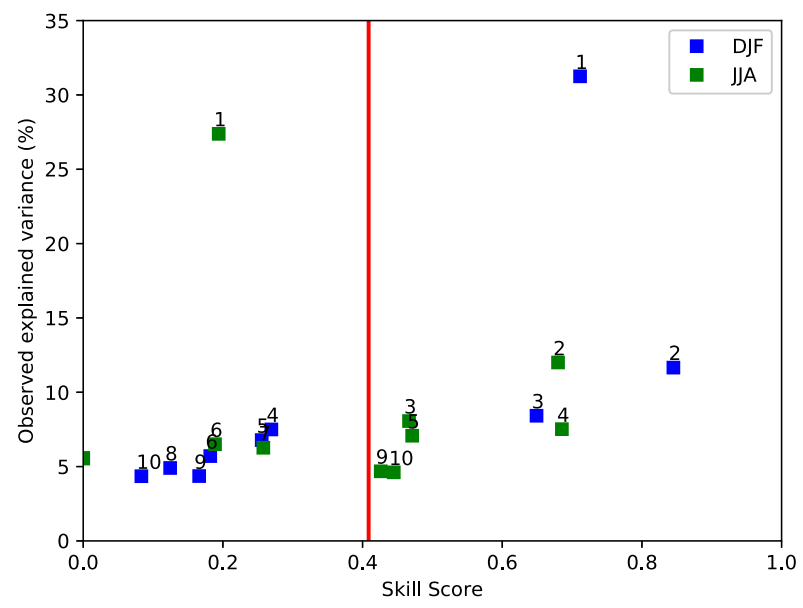

FIG. 2. Percentage of variance explained by the first 10 observed modes ( $y$ axis) and the skill score obtained by the MMEM modes ( $x$ axis) for DJF (blue) and JJA (green) 200-hPa geopotential height. The numbers denote the mode while the red line denotes the skill score significance threshold at the $95 \%$ confidence level.

skills for the first and second modes are higher than 0.85 (0.90 and 0.86) while PCC skill for mode 3 is lower (0.69). In particular, the change of sign between low and high latitudes observed in these three modes is well captured (Figs. 3b,f,j). However, MMEM fails in reproducing the wavelike structure of the third mode, describing instead a more zonally symmetric structure. Figure 4 shows that the MMEM predicts well the interannual variability associated with the three PCs, with TCC skills of $0.56,0.82$, and 0.60 , respectively. In particular, PC extreme values for the second mode are remarkably well predicted.

The REOF applied to the MMEa allows the analysis of the main modes of variability associated with the differences between the mean of each model and the MMEM. The modes obtained with the MMEa show a similar behavior than those obtained through the MMEM. The percentage of variance explained by each mode is $49 \%, 22 \%$, and $15 \%$, respectively. The corresponding PCC as well as TCC values for the three modes resulting from MMEa are high (Table 2). The MMEa first mode (Fig. 3c) shows the characteristic annular distribution although, in contrast to observations, negative anomalies span up to $60^{\circ} \mathrm{S}$, and positive anomalies at midlatitudes are shifted. On the other hand, the second mode (Fig. 3g) shows at the tropics larger positive anomalies than for the observed mode. Moreover, at middle and high latitudes, anomalies are weaker and, in some cases, such as near the Antarctic Peninsula, anomalies are negligible. The third mode (Fig. 3) is similar to that obtained from MMEM, although negative anomalies are weaker. The PCs associated with the 
a)

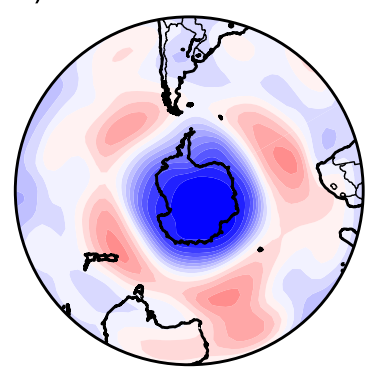

e)

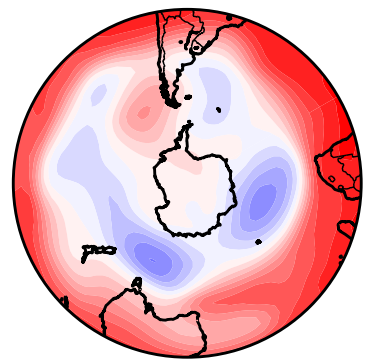

i)

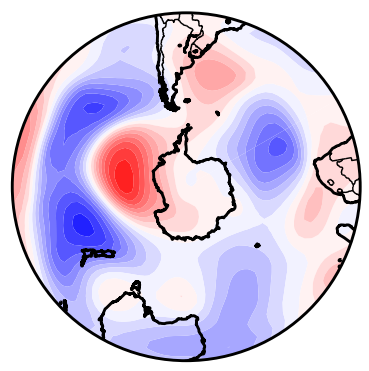

b)

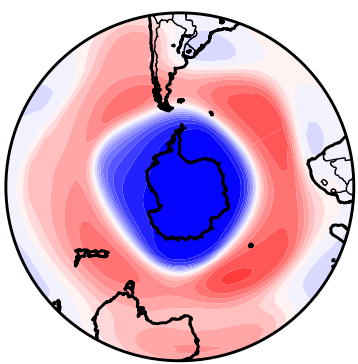

f)

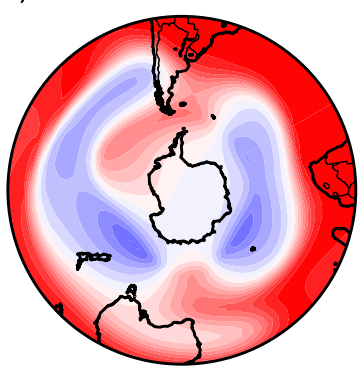

j)

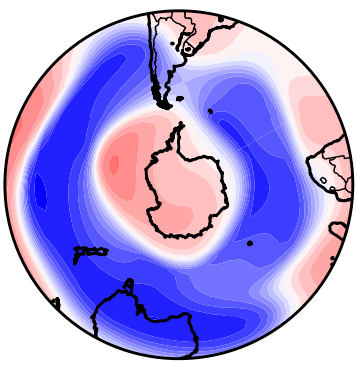

c)

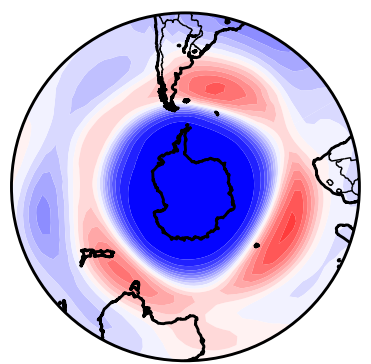

g)

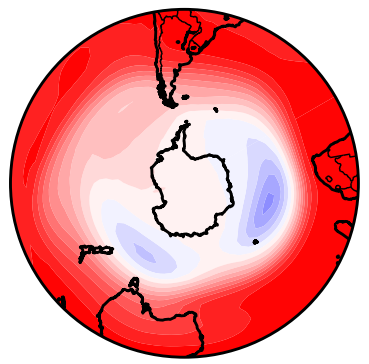

k)

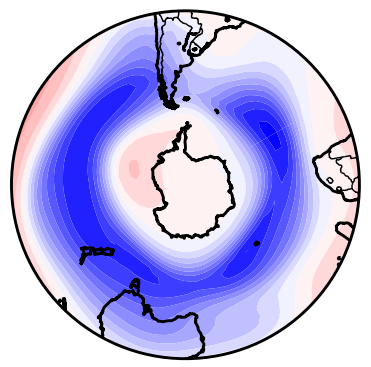

d)

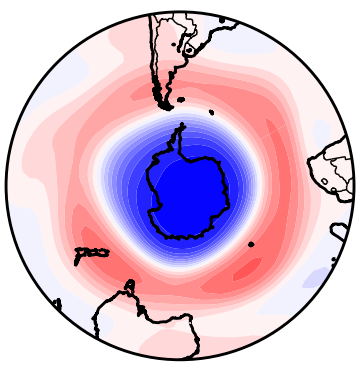

h)

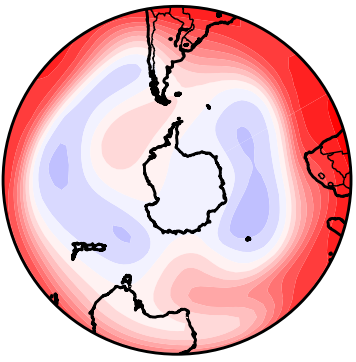

l)

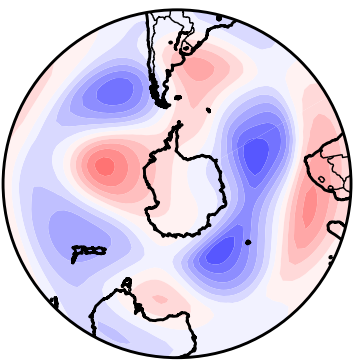

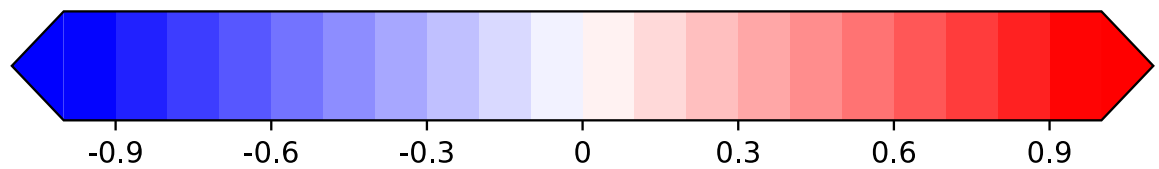

FIG. 3. Spatial patterns (REOFs) of 200-hPa geopotential height in DJF for the (a) first, (e) second, and (i) third mode for observations; (b) first, (f) second, and (j) third mode for MMEM; (c) first, (g) second, and (k) third mode for MMEa; and (d) first, (h) second, and (l) third mode for SMMEa.

modes derived from MMEa not only allow comparison with those derived from observations but also facilitate the assessment of the dispersion among the PCs of each individual member. The second PC presents the lowest dispersion among members. The first PC, on the other hand, shows the highest dispersion among members, while that for the third PC is also considerable (Figs. 4a-c).

The variability associated with the noise is studied through the REOF analysis applied to the SMMEa. These modes agree with those obtained from the observations. The variance explained by each mode (37\%, $8 \%$, and $5 \%$, respectively) is lower that the percentages obtained with the MMEM and MMEa. On the other hand, the corresponding PCC for the three leading modes are even higher than those for the MMEa modes (Table 2). SMMEa better reproduces the monopole characterizing the first mode, but it depicts less defined and more zonally extended midlatitude positive anomalies (Fig. 3d) than other ensembles. The position and intensity of the anomalies corresponding to the second mode from SMMEa match well with those derived from observations (Fig. 3h). The third mode (Fig. 31) is very well represented, including the location and amplitude of the corresponding wave train centers, improving the performance of the other prediction ensembles. Although significant TCC values were obtained from SMMEa for the three modes, as was previously reported 

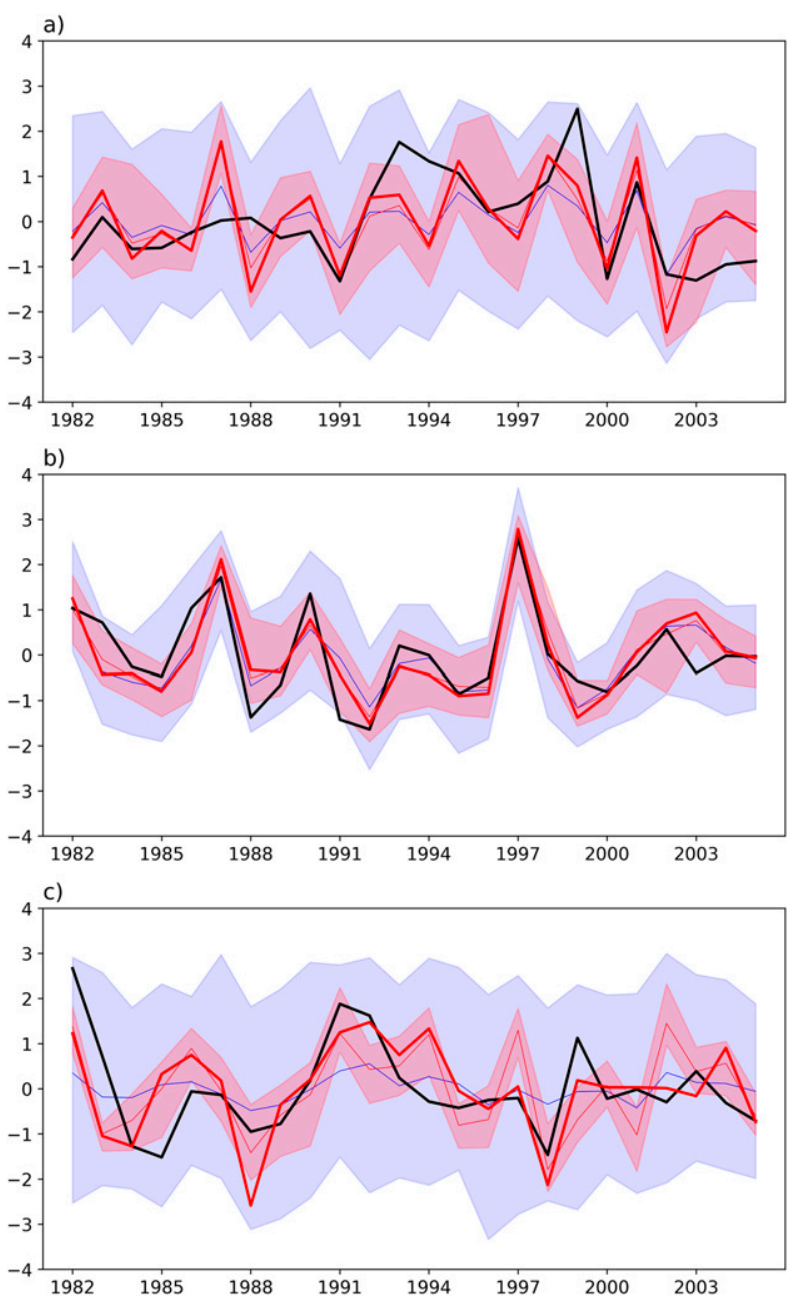

FIG. 4. Temporal series (PCs) for the (a) first, (b) second, and (c) third mode of variability of the 200-hPa geopotential height in DJF for observations (black line), MMEM (thick red line), MMEa ensemble members (red shading) and its mean (thin red line), and SMMEa ensemble members (blue shading) and its mean (thin blue line).

for the MMEa, the first and third ones show high dispersion while the agreement between members in the second one is considerably high.

Figure 3 also allows the comparison of the structure of the modes obtained with a different ensemble configuration. The modes obtained with the MMEa present a more zonal structure than the MMEM and therefore they are less correlated with those observed, which implies that the observed pattern cannot be completely recovered from the predictions if the mean of each model is used to compute the modes. Moreover, the modes obtained with the SMMEa can reproduce the wave structure observed, especially in mode 3 , impacting the values of the PCC obtained (higher than those from the MMEa).
TABLE 2. Correlations of DJF 200-hPa geopotential height observed REOFs and PCs against the different ensemble constructions considered.

\begin{tabular}{lccc}
\hline \hline & MME & MMEa & SMMEa \\
\hline REOF1 & 0.90 & 0.82 & 0.94 \\
REOF2 & 0.86 & 0.85 & 0.88 \\
REOF3 & 0.69 & 0.65 & 0.79 \\
PC1 & 0.56 & 0.52 & 0.55 \\
PC2 & 0.82 & 0.86 & 0.83 \\
PC3 & 0.60 & 0.48 & 0.57 \\
\hline
\end{tabular}

Very few works have previously performed an assessment of climate models in reproducing these modes of tropospheric circulation variability. Grainger et al. (2011) used five AGCMs from the Climate of the Twentieth Century (C20C) Project and showed that the models can reproduce the first three modes of variability derived from $500-\mathrm{hPa}$ geopotential heights with similar skills than those obtained in this work. Although in Grainger et al. (2011) the first mode is the best reproduced one, followed by the third one, the variance explained by the simulated modes is lower than the one explained by the observed modes. It is worth pointing out that the models used by Grainger et al. (2011) are atmospheric models forced with the same SST field, and therefore the modes obtained only capture the models' response to the common SST forcing, while our analysis is based on coupled models. In addition, Grainger et al. (2011) only assessed the performance of each model separately instead of the multimodel ensemble. More recent works showed that models from phases 3 and 5 of the Coupled Model Intercomparison Project (CMIP3 and CMIP5) are also able to reproduce these modes in their historical experiments (20C and Historical, respectively; Grainger et al. 2013, 2014, and references therein) although the dispersion among models is large. The comparison of the modes obtained with the MMEa with the CMIP "slow internal" modes from Grainger et al. $(2013,2014)$ reveals that the annular structure in the first mode is better captured by CMIPs models than by CHFP models while the second mode is reproduced with similar ability by the simulations of both datasets. Some differences in the pattern obtained as the third mode are found between our work and Grainger et al. $(2013,2014)$. This can be related to the fact that, unlike previous works, we analyzed rotated EOFs.

\section{2) SOURCES OF PREDICTABILITY}

Given that the first three modes are skillfully forecast by the MMEM, we then explore their sources of predictability associated with SST anomalies. The analysis was made by computing correlation maps between PCs 
a)

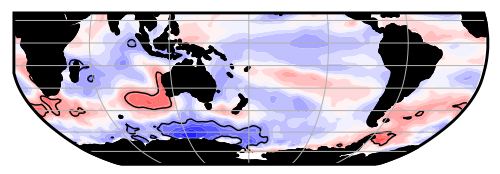

d)

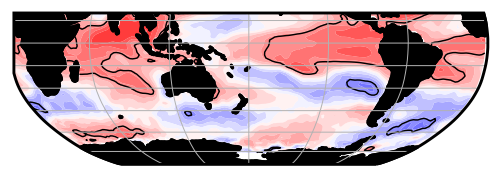

g)

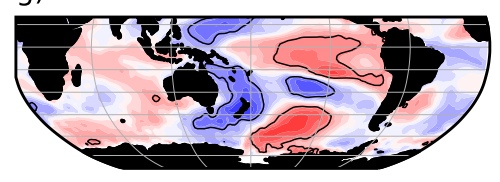

b)

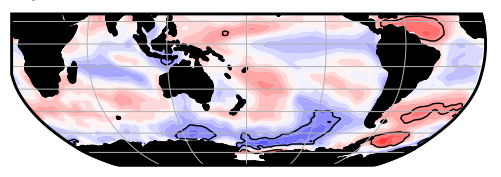

e)

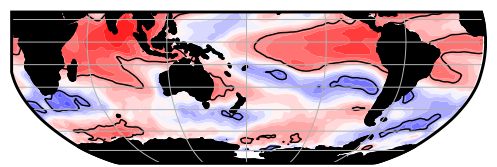

h)

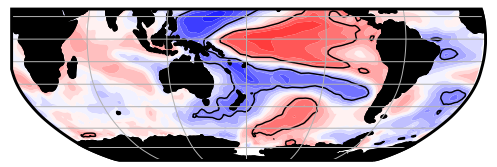

c)

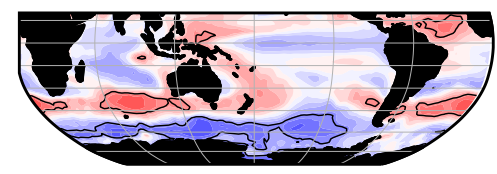

f)

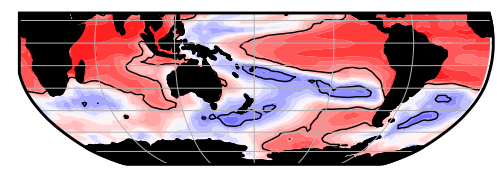

i)

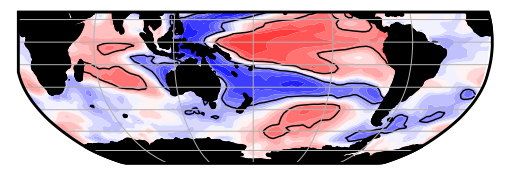

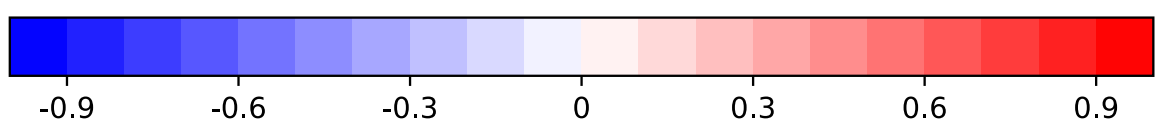

FIG. 5. Correlations between the three predictable modes of DJF 200-hPa geopotential height and DJF SST for (a),(d),(g) observed modes against observed SST, (b),(e),(h) MMEM modes against observed SST, and (c),(f),(i) MMEM modes against forecast SST. Each row corresponds to each predictable mode. The thin line marks the significance threshold at the $95 \%$ confidence level.

from the three leading observed modes and contemporaneous observed SST, as well as correlation maps between forecast PCs and contemporaneous observed SST, and correlation maps between forecast PCs and forecast SST.

Correlations for the first mode show larger values at the extratropics than at the tropics (Figs. 5a-c). The first observed mode is significantly correlated with SST anomalies with a negative sign only over small areas at the tropics and south of Australia-New Zealand sector, while significant positive correlations are observed near the Antarctic Peninsula, off South Africa, and in western Australia (Fig. 5a). Correlation is negative but nonsignificant over the central tropical Pacific and Indian Oceans. Correlations between observed SST anomalies and the first mode derived from MMEM display a similar spatial distribution (Fig. 5b), although the negative correlations at polar latitudes are shifted eastward with respect to observed correlations, while positive correlations over the Antarctic Peninsula are significant and higher than those for observations. The correlation between forecast mode and forecast SST (Fig. 5c) also displays a similar spatial distribution, with values overall higher than those between forecast mode and observed SST. At middle latitudes, correlations between forecast mode and forecast SST are mostly positive and significant, peaking at the Indian and Atlantic Oceans, while at polar latitudes they are negative and significant.
Correlation between observed second mode and observed SST anomalies (Fig. 5d) shows the characteristic positive-phase ENSO-like pattern. Positive and significant correlations are discernible over the central-eastern tropical Pacific, surrounded by negative values distributed in the typical horseshoe pattern (but with significant values only in small areas). Positive and significant correlations extend along the tropical Atlantic and Indian Oceans, while at the midlatitudes negative values dominate but they are mostly nonsignificant. Correlations between forecast second mode and observed SST match those of the observed mode, although values are higher, especially over the Indian Ocean (Fig. 5e). Correlations between forecast second mode and forecast SST anomalies (Fig. 5f) show that MMEM is able to reproduce the link between ENSO and the mode, although values are higher and positive correlations at the tropical Atlantic and Indian Oceans span a wider band of latitudes.

Significant positive correlations between observed third mode and observed SST are found at tropical central Pacific (Fig. 5g). Over the Indian Ocean, correlations are positive, whereas over the Atlantic they are negative, but none of them is significant. At the extratropics, the correlations are like those obtained for the second mode although over the Pacific (Atlantic) they are stronger and significant (weaker and nonsignificant). Correlations between the forecast third mode and observed SST anomalies (Fig. 5h) are similar to those obtained for the observed mode, but slightly stronger over 
the Pacific. As was pointed out for previous modes, correlations between the forecast third mode and forecast SST show higher values than for observations, especially over the tropical Atlantic and Pacific Oceans (Fig. 5i).

The regions where correlations between the observed first mode and observed SST maximize do not agree with those obtained by previous work (Grainger et al. 2011). This can be due to differences in the period considered and to the strong decadal variability that influences SAM activity (Silvestri and Vera 2009). Also, a significant SAM linear trend has been described in the second half of the twentieth century (Arblaster and Meehl 2006), which has been removed in this work, but not in Grainger et al. (2011). The correlations between the observed second and third mode and observed SST anomalies have been documented by many previous works (e.g., Frederiksen and Zheng 2007; Vera et al. 2004) and they agree in general with those obtained here. However, very few works show how models reproduce this link. Grainger et al. (2013) found that CMIP3 models from the 20C experiment are able to reproduce the relationship between these modes and SST. The authors attributed this to the influence that ENSO has over these modes.

\section{b. $J J A$}

\section{DESCRIPTION OF VARIABILITY AND IDENTIFICATION OF PREDICTABLE MODES}

We repeat the analysis done for DJF but for JJA. Figure 1 shows in the right column the variance for JJA of 200-hPa geopotential heights from observations, MMEM, and the MMEa, normalized by the variance averaged over the domain. As for DJF, major observed variability is found at polar latitudes over Bellingshausen-Amundsen and Ross Seas where variance doubles the spatial mean variability (Fig. 1b). Another maximum is observed over eastern Antarctica, smaller than the previous one and smaller than that for DJF. Over the central South Pacific Ocean a local maximum is observed, slightly higher than spatial mean variability. On the other hand, the variance for the MMEM presents a different distribution, with maximum values located over the central South Pacific Ocean and over the Ross and Amundsen-Bellingshausen Seas (Fig. 1d). Also, over southern Africa and the South Atlantic Ocean, variability is somewhat higher than the spatial mean variability. Over Antarctica, the local maximum observed is not present in the variance resulting from MMEM whereas it is evident in that obtained from MMEa (Fig. 1f). Furthermore, the variance from MMEa in eastern Antarctica is bigger than that observed. At the tropics and midlatitudes the ratio of variances is less than one in most of the domain.
We also evaluated the performance of the MMEM in capturing the main modes of variability of the 200-hPa geopotential heights in JJA by computing the EOF analysis and rotating the modes, as was done for DJF. Only the first mode of variability is statistically distinguishable from the rest of the modes. However, the skill in reproducing it is not significant (due to the inability of the MMEM to reproduce the associated time series) while it is for the next four modes (Fig. 2, green dots). In this sense, it is worth pointing out that mode 2 and mode 4 present as good skill as DJF predictable modes. Therefore, we consider that for JJA the second and fourth modes of variability are predictable modes due to their good representation by models and for being the JJA counterpart of DJF mode 3 and mode 2 (see below). Nevertheless, we describe the main characteristics of the first five modes and we show how models reproduce them. The five modes capture about $62 \%$ of the observed variability and $83 \%$ of the predicted variability over the domain.

Figure 6 is similar to Fig. 3 but for the first five REOFs of JJA. The first observed mode (Fig. 6a) accounts for about $27 \%$ of total variance and, as in DJF, it is associated with SAM. The negative monopole observed in the pole is accompanied at middle latitudes by positive anomalies embedded in a wave- 4 pattern (instead of the wave- 3 pattern like in DJF), and weaker negative anomalies at the tropics. The associated PC time series (Fig. 7a) shows low variability in the first half of the period while it increases at the end. The second mode accounts for $12 \%$ of the observed variance and shows alternating positive and negative anomalies extending over the western Pacific Ocean, the South Pacific Ocean, and the western South Atlantic Ocean (Fig. 6e), resembling the PSA2. The third mode (Fig. 6i) accounts for about $8 \%$ of the observed variance and presents high positive anomalies over the eastern Pacific Ocean, the South Atlantic Ocean, and the Indian Ocean while weak negative anomalies are observed south of Australia and over the Antarctic Peninsula. This mode does not have a counterpart in the modes analyzed for DJF. The associated time series shows high year-to-year variability (Fig. 7c). The fourth mode (Fig. 6m) accounts for $7.5 \%$ of the observed variability and shows at the tropics large negative anomalies zonally extended while at the extratropics and polar latitudes traces of a wave train pattern are present. This mode resembles that known as PSA1 (similar to the second mode observed in DJF). Accordingly, the corresponding PC time series maximizes in some ENSO years like 1988, 1989, 1998, and 2000 (Fig. 7d). The fifth mode accounts for about 7\% of the observed variance and has positive anomalies at the tropics and midlatitudes, conforming to a wave-5 
a)

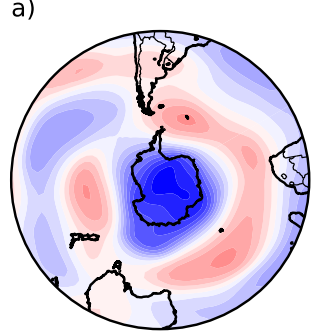

e)

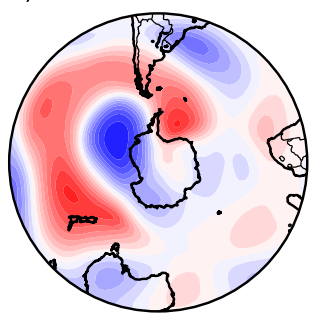

i)

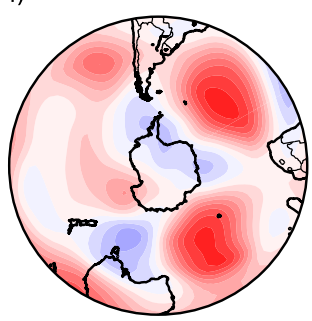

m)

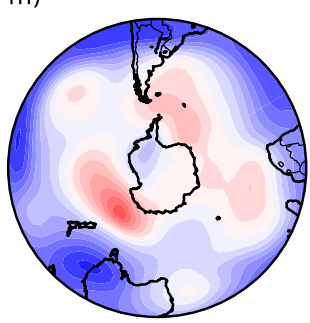

q)

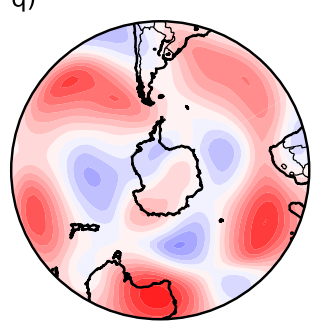

b)

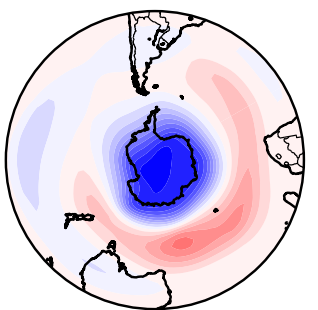

f)

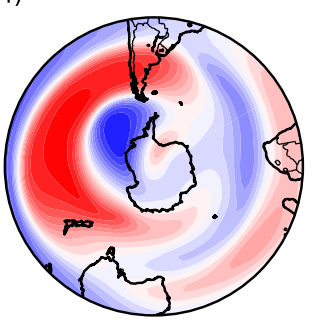

j)
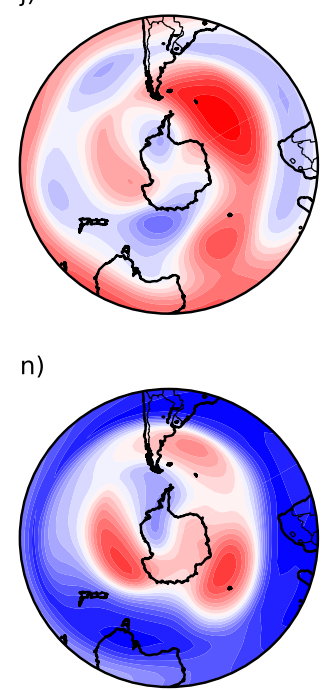

r)

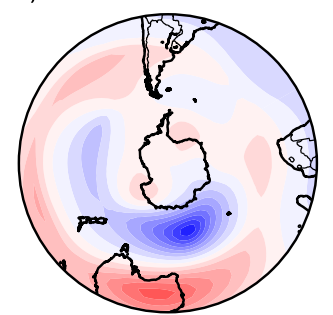

c)

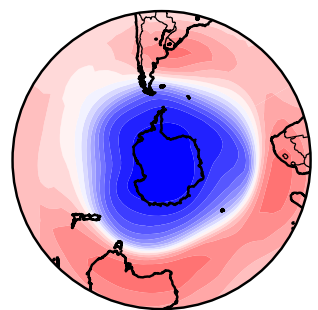

g)

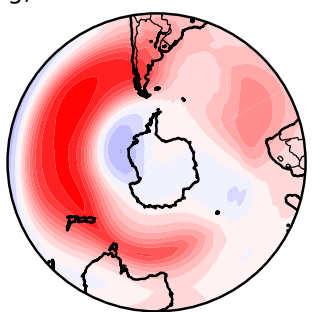

k)

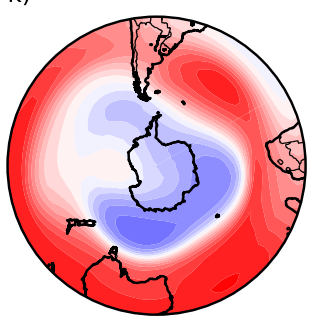

o)

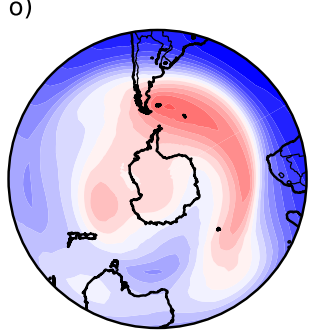

s)

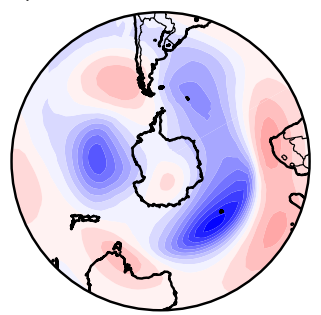

d)

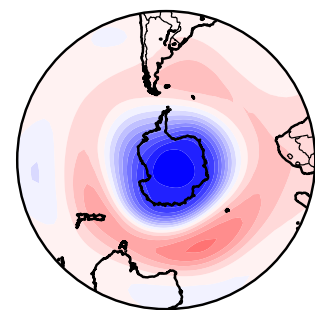

h)

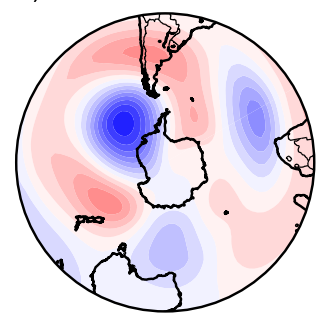

I)
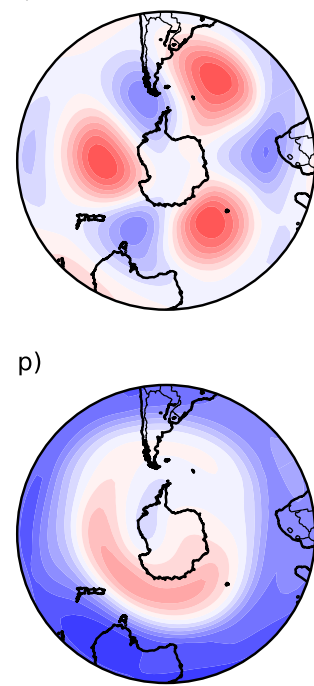

t)

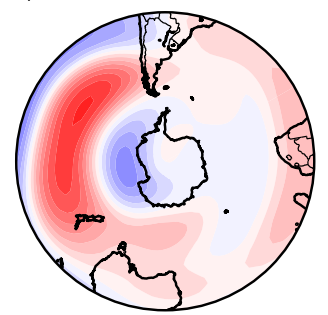

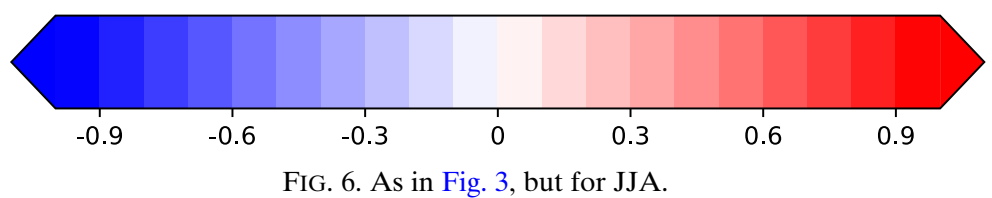



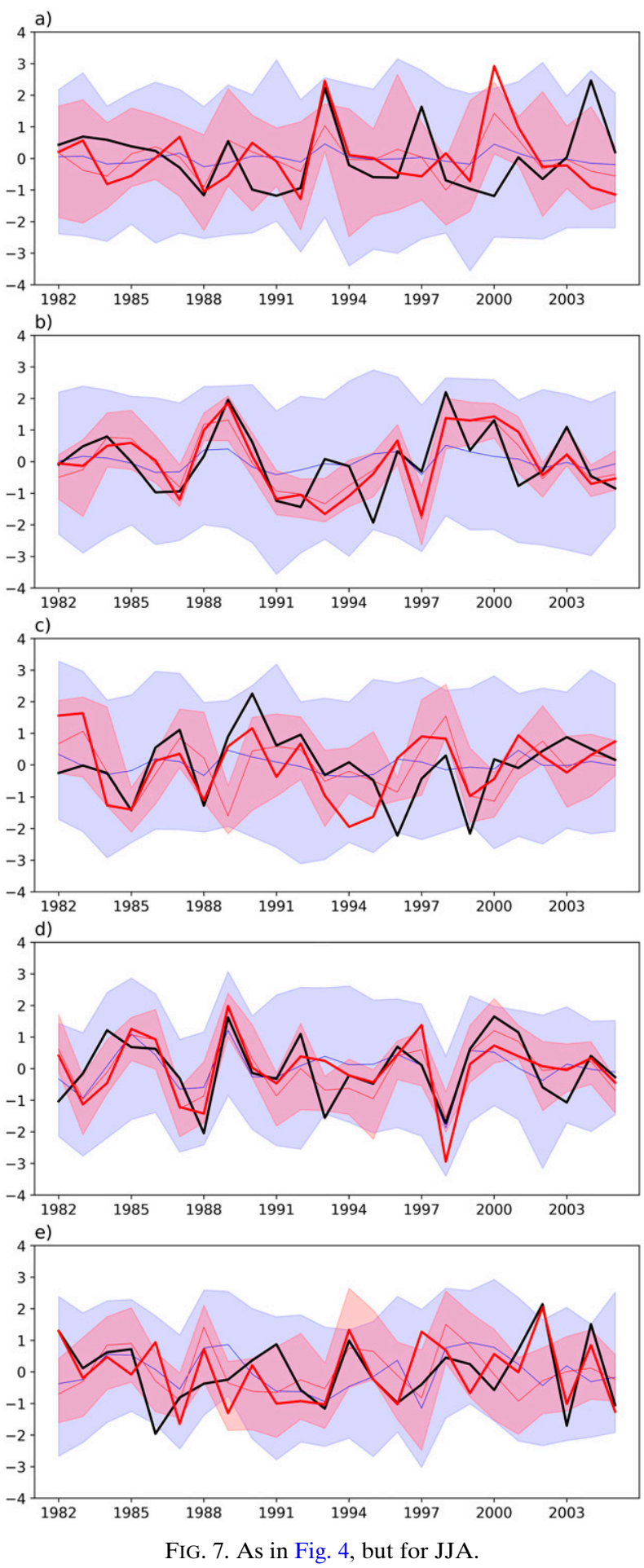

pattern, while at high latitudes anomalies are mostly negatives (Fig. 6q). The associated time series presents a similar degree of variability as the previous modes (Fig. 7e).

As was mentioned before, the MMEM can predict well the spatial distribution of the five modes, but it fails
TABLE 3. As in Table 2, but for JJA.

\begin{tabular}{lccc}
\hline & MME & MMEa & SMMEa \\
\hline REOF1 & 0.92 & 0.66 & 0.92 \\
REOF2 & 0.71 & 0.59 & 0.70 \\
REOF3 & 0.53 & 0.48 & 0.63 \\
REOF4 & 0.74 & 0.69 & 0.77 \\
REOF5 & 0.41 & 0.50 & 0.27 \\
PC1 & 0.04 & 0.13 & 0.13 \\
PC2 & 0.65 & 0.63 & 0.63 \\
PC3 & 0.40 & 0.41 & 0.21 \\
PC4 & 0.62 & 0.69 & 0.62 \\
PC5 & 0.53 & 0.12 & -0.04 \\
\hline
\end{tabular}

in reproducing their associated time series. The percentage of variance explained by each mode is $27 \%, 16 \%$, $12 \%, 24 \%$, and $3 \%$, respectively. The highest PCC values are obtained for the first, second, and fourth mode. On the other hand, TCC is not significant for the first mode (justifying why this mode is considered nonpredictable). Furthermore, the second and fourth modes show high TCC values. In the case of the second mode, this is explained by the good performance of the MMEM in years with large mode amplitude (Fig. 7b). The time series for the fourth mode shows a good representation of the observed mode year-to-year variability including the amplitude of maximum values (Fig. 7d).

The PCC values obtained from the MMEa are high (above 0.6) and fairly similar to those from MMEM (Table 3). As for DJF, in all the modes analyzed the signs of the anomalies are well forecast although they are elongated in the zonal direction. On the other hand, despite the TCC values being low, as was reported for the MMEM, the modes identified with PSA-like patterns present significant TCC values and the lowest dispersion between ensemble members. The percentage of variance explained by these modes is $46 \%, 12 \%, 18 \%$, $9 \%$, and $6 \%$, respectively. The analysis of the modes obtained from the SMMEa shows high PCCs against observed modes. As in DJF, the modes associated with the SMMEa better represent the wave trains observed in midlatitudes for most of the modes. As for the other ensemble approaches, the TCCs from SMMEa, obtained with the mean of all member time series, present significant correlation only for the second and fourth modes. In agreement with DJF, the first mode explains a percentage of variance much higher than the rest of the modes $(28 \%, 6 \%, 6 \%, 9 \%$, and $6 \%$, respectively).

As was documented for DJF, the modes obtained with the MMEa are more zonally oriented than those obtained with the MMEM. In addition, the wave structure that characterize PSA2 (second mode) is well captured by the SMMEa but not by the MMEM or the MMEa. 
a)

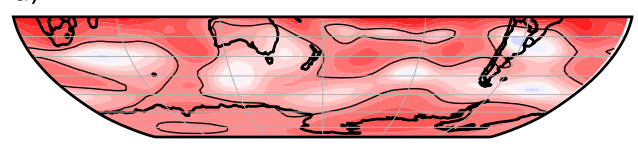

c)

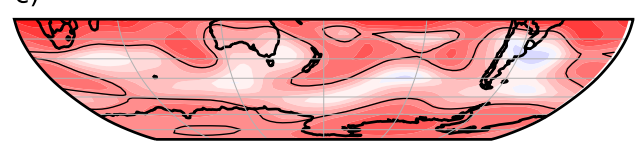

e)

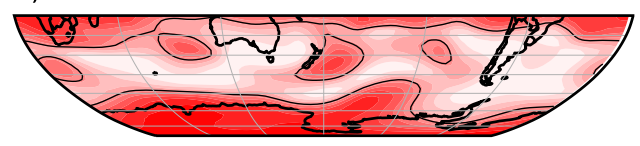

b)

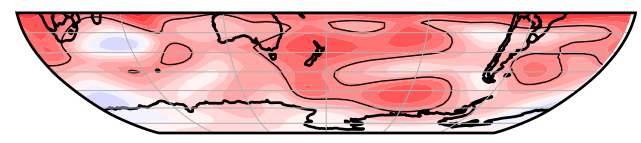

d)

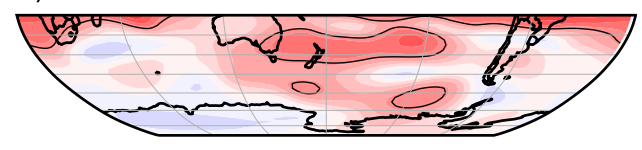

f)

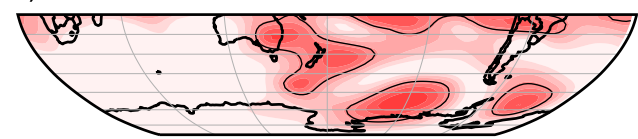

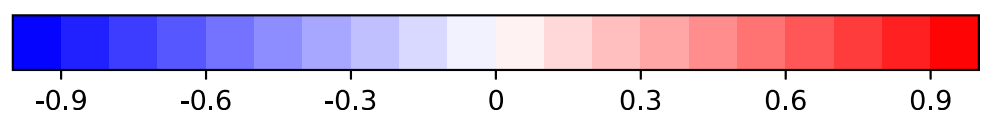

FIG. 8. Correlation coefficient between observed 200-hPa geopotential height for (a) DJF and (b) JJA and MMEM 200-hPa geopotential height (skill); (c) DJF and (d) JJA MMEM 200-hPa geopotential height reconstructed with predictable modes; and (e) DJF and (f) JJA and observed 200-hPa geopotential height reconstructed with predictable modes (potential predictability). The dotted line denotes the $95 \%$ confidence limit.

In agreement with our findings, previous works with a focus on the circulation in the SH (e.g., Grainger et al. 2011, 2013, 2014) showed that model performance in reproducing the leading modes for JJA is lower than that for DJF, using both AGCMs from the 20C project or CMIP3 and CMIP5 models, while the dispersion among models described in those works is, as in this work, higher in JJA than in DJF. However, the percentage of variability explained by the main modes from CMIPs models is similar to that obtained with observations, in contrast to our work, in which CHFP modes overestimated the relative contribution of the modes to the total variability. On the other hand, Grainger et al. (2014) used CMIP5 models to study the sensitivity of the simulated modes to the number of models participating in the ensemble. They found significant changes in the performance of the MMEM with the increase of the number of models included in the ensemble, suggesting that it is crucial to have more than 10 models in the ensemble to diminish the variability in the performance of the MMEM in this season. Therefore, as our work was made with more than 10 models, the poor performance of the model ensemble in JJA might not be related to the ensemble size.

In contrast to the results obtained for DJF, the correlations between PCs and SSTs for JJA are mostly nonsignificant (see the online supplemental material). Mo (2000) shows that in this season PSA1 pattern variability exhibits a ENSO-like correlation distribution with SSTs, although values are weaker than for DJF. On the other hand, Mo (2000) also shows that the correlations between PSA2 and SSTs are mostly nonsignificant. In agreement, the correlations between the observed second and fourth mode and observed SSTs are almost negligible in this work. Regarding the second forecast mode, the overall spatial distribution of correlations with observed SSTs is well captured but with values larger than observed. Finally, the correlation between forecast second mode and forecast SST looks like an intense ENSO-like pattern, not identified in the observed correlations. Regarding the fourth mode, the correlation between the forecast mode and the observed SST reproduces well the observed pattern. Instead, the correlations between forecast fourth mode and forecast SST show a negative ENSO-like pattern, although with values at the central Pacific shifted southward.

\section{c. Prediction skill and potential predictability}

In this section we describe the current level of prediction skill achieved by the MMEM prediction and provide a discussion about the predictability that can be attained by the predictable modes. As in Lee et al. (2011), we assume that the leading modes are more predictable than the higher modes. Consequently, we decompose the observed and forecast fields into the predictable and unpredictable parts. The predictable part is reconstructed with the linear combination of the predictable modes while the unpredictable part is calculated by linearly combining the unpredictable modes. Then, the potential predictability is estimated by the 
TCC computed between the observed total field and the observed predictable part. Thus, if models could perfectly forecast the observed predictable modes it would represent the achievable prediction skill.

Figure 8 shows the prediction skill of the MMEM for DJF and JJA, together with the prediction skill obtained with the forecast predictable modes and the potential predictability. As was documented by Osman et al. (2016), prediction skill in DJF is higher at the tropics, reaching 0.9 in some regions, decreases at midlatitudes to nonsignificant values, and increases again at the polar latitudes, peaking over the Amundsen-Bellingshausen Sea (Fig. 8a). The skill attained by the MMEM comes mainly from the prediction skill resulting from the first three forecast predictable modes (Fig. 8c) while the forecast nonpredictable modes contribute positively but nonsignificantly to the skill at midlatitudes (not shown). The decomposition of the prediction skill in that associated to each mode (not shown) shows that the skill associated with the first (second) mode contributes uniformly to the skill at the polar (tropical) regions while that associated with the third mode contributes in some regions at the midlatitudes, like the Pacific and Indian Oceans. The potential predictability (Fig. 8e) shows that variability over the extratropics, especially over eastern Antarctica, is highly predictable due to the first three modes. However, the middle latitudes, and especially South America, are by far less predictable than the polar latitudes. The prediction skill achieved by the MMEM is lower than that obtained with the forecast predictable modes at most of the polar zone but it is higher than potential predictability in some parts of the tropics and midlatitudes. On the other hand, prediction skill in JJA is highest at the tropics but lower than in DJF and decreases poleward, maximizing at midlatitudes over the Pacific Ocean at $35^{\circ}$ and $65^{\circ} \mathrm{S}$ (Fig. 8b). If we compare this skill with that achieved by the forecast predictable modes it can be concluded that a large part of the prediction skill comes from the prediction skill of those modes. On the other hand, the potential predictability is significant in the tropical central and eastern Pacific Ocean while it presents a wave pattern emanating from eastern Australia toward the Pacific and South Atlantic Oceans in the middle and high latitudes.

Very few works have assessed the predictability of the main modes of variability of the $\mathrm{SH}$ circulation. Of particular interest is the work of Zheng and Frederiksen (2007), who developed a statistical prediction of the SH circulation at $500 \mathrm{hPa}$ by forecasting its main modes of variability for DJF and JJA. They found that skill is higher in the tropics than in the polar regions and in DJF than in JJA, results that agree with our work. It should be noted that in both works, modes related to SST variability in tropical regions are better forecast in both seasons. Furthermore, in Zheng and Frederiksen (2007) the SAM contributes significantly to the skill achieved by the statistical model in DJF but does so only marginally in JJA. Finally, modes associated with variability in the tropical SSTs, like PSAs, contribute to most of the skill observed in tropical latitudes.

\section{Associated surface climate anomalies}

In this section we analyze the climate anomalies associated with the predictable modes in both observations and the MMEM. Figure 9 shows correlation maps for DJF between 2-m air temperature and precipitation against the predictable modes obtained from observations (first and third column, respectively) and MMEM predictions (second and fourth column, respectively). In observations, temperature correlations associated with the first mode are characterized by negative values over most of the $\mathrm{SH}$, being significant at the pole over the eastern Maritime Continent and southern Australia and Africa, while positive values are observed over the southern Indian and Atlantic Oceans (Fig. 9a). The MMEM captures the negative correlations although weaker than observed, and therefore they are mostly nonsignificant (Fig. 9b). Correlations between the first observed mode and precipitation are mostly nonsignificant (Fig. 9c) while correlations between the MMEM first mode and precipitation are similar to those observed (Fig. 9d).

The observed second mode is correlated positively with temperature at tropics, maximizing over northern South America and the Indian Ocean (Fig. 9e). In the extratropics, correlations are negative off of South Africa, Australia, southern South America, and over the central Pacific Ocean, while at the polar regions correlations are positive but weak. MMEM captures with high fidelity the observed correlations against temperature although with values slightly higher than observed (Fig. 9f). Correlations between the observed second mode and precipitation are characterized by the typical ENSO signal with positive values over the eastern Pacific Ocean and the northern Indian Ocean, and negative ones over the Maritime Continent and northern South America (Fig. 9g). As for temperature, MMEM represents well the correlation distribution but values are larger than the observed counterpart, especially over the western Pacific Ocean and the Maritime Continent (Fig. 9h).

Correlations between temperature and the observed third mode are characterized by large positive values over the eastern and South Pacific Ocean and weak positive ones over northern South America and the Indian Ocean (Fig. 9i). Negative values are present over 
a)

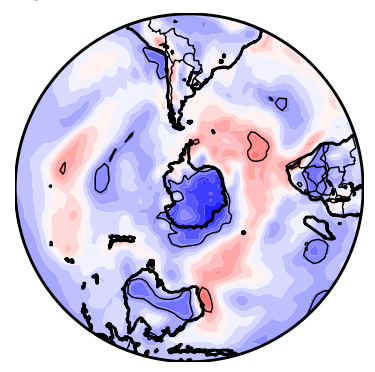

e)

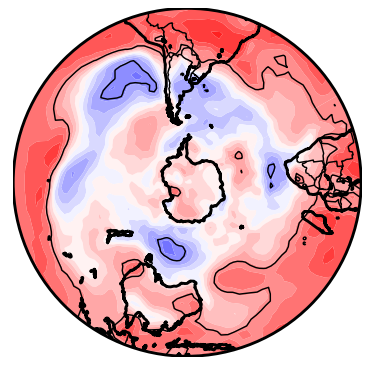

i)

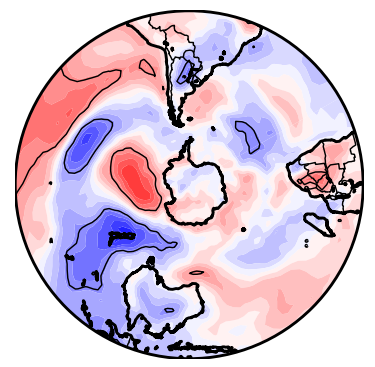

b)

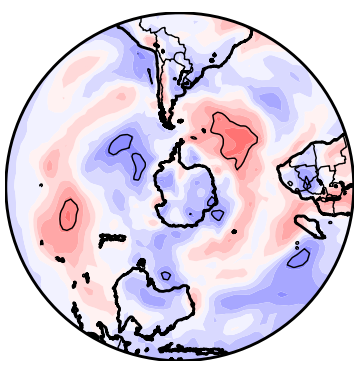

f)

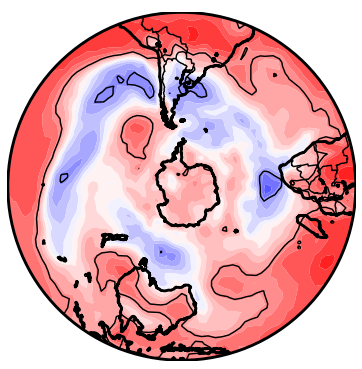

j)

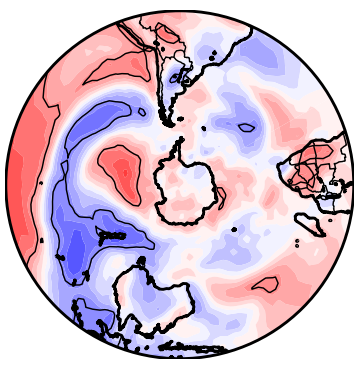

c)

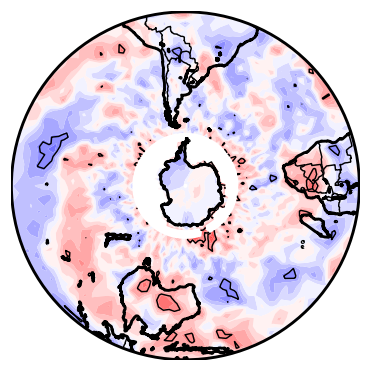

g)

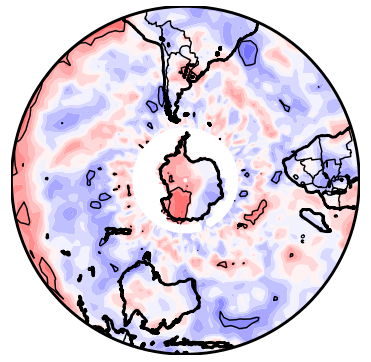

k)

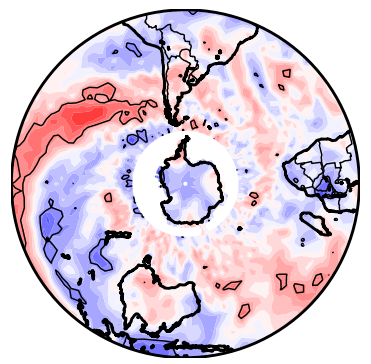

d)

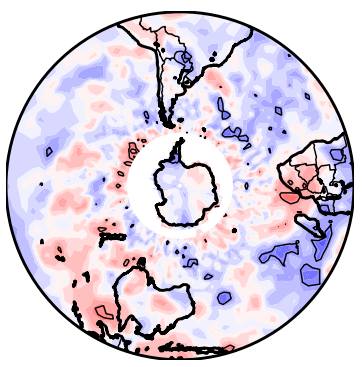

h)

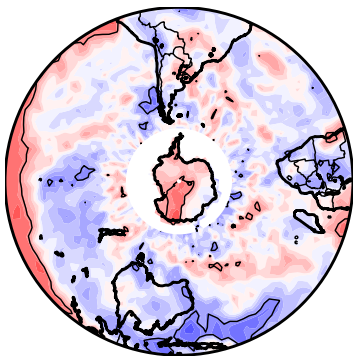

l)

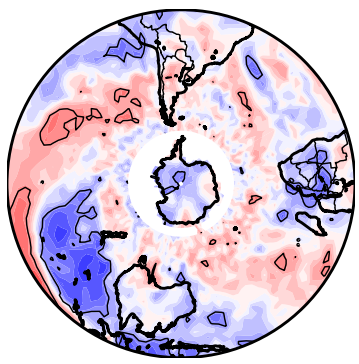

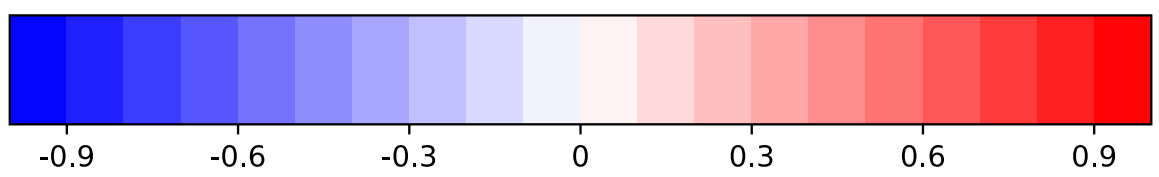

FIG. 9. Correlation between the predictable PCs for observed 200-hPa geopotential heights in DJF and (a),(e),(i) observed 2-m temperature and $(\mathrm{c}),(\mathrm{g}),(\mathrm{k})$ precipitation, and correlation between the predictable PCs for MMEM 200-hPa geopotential height in DJF and (b),(f),(j) observed 2-m temperature and (d),(h),(l) precipitation. Each row corresponds to each predictable mode. The dotted line denotes the $95 \%$ confidence limit.

the Maritime Continent, the western and south-central Pacific Ocean, the southern Atlantic Ocean, and southern South America. The MMEM succeeds in predicting in general the pattern but with values slightly different from those observed, especially over the central Pacific Ocean (Fig. 9j). Precipitation correlations in association with the observed third mode (Fig. 9k) are positive over the central tropical Pacific Ocean and negative to both sides over northern South America and the Maritime Continent. In the extratropics correlations are positive over the eastern Pacific Ocean, southern South America, the Indian Ocean, and Australia while they are negative over the western Pacific Ocean and southern Africa. However, correlations are significant mostly over the Pacific Ocean. The MMEM overestimates the negative correlations in most of the domain whereas it underestimates (overestimates) the positive correlations in the extratropics (tropics) (Fig. 91).

In JJA (not shown), the second mode is correlated significantly with temperature in a small region of the ocean. This correlation is positive in the western-central Pacific Ocean and is also captured by the MMEM. On the other hand, the fourth mode is not significantly correlated with temperature in the observations or the MMEM. Finally, both modes do not present significant correlation with precipitation. 


\section{Conclusions}

In this work an analysis of the predictability of the Southern Hemisphere tropospheric circulation is performed for DJF and JJA, through the representation of the main modes of variability. A rotated EOF analysis was performed for the $200-\mathrm{hPa}$ geopotential height anomalies derived from observations and from the lead-1 MMEM forecast obtained from the WCRP-CHFP database. The ability of the MMEM in forecasting the observed modes of variability was assessed, as well as the potential predictability achieved by these modes.

We found that the first three leading modes of variability in DJF are predictable. These modes are well known as the southern annular mode and the PacificSouth America patterns. The MMEM is able to represent better their spatial distribution than their temporal evolution. The exception is mode 2, which is strongly linked to ENSO and is also well represented temporally. In contrast, the MMEM exhibits in JJA a poor performance in reproducing the first mode of variability while it very well reproduces the second and fourth mode. The latter and the fact that the second and fourth modes are related to dynamical processes observed in the circulation and documented in previous works led us to conclude that these modes are predictable.

Different ensemble constructions were assessed to exploit the large amount of information available as well as to evaluate differences in models response. We first averaged all models to represent the mean model response (MMEM), then we constructed the ensemble with the anomaly of each model ensemble mean with respect to the MMEM to characterize the differences in the model response (MMEa), and finally we constructed the ensemble with the anomalies of all ensemble members from all models with respect to the ensemble mean of each model to represent the noise (SMMEa). It was found that the MMEM is able to reproduce the sign of the teleconnections that characterizes PSA patterns at middle and high latitudes although the wave train structure is somewhat diffuse, especially for PSA2. In addition, the modes obtained with the MMEa are more zonally oriented than their counterparts obtained with the MMEM, which means that the main differences between the response associated with each model and the response common to all of them are mainly zonal. Therefore, neither the variability associated to the mean model response nor the variability associated with differences in model response reproduces the wave structure present in observed modes. In this sense, the modes associated with noise (SMMEa) better represent the variability at midlatitudes obtained with the observed modes. Moreover, exploiting the full ensemble available in this study leads to a PSA2 pattern remarkably similar to that observed. These results are of particular importance since previous works have analyzed the variability in the $\mathrm{SH}$ depicted by different datasets but just focused on the ensemble mean of each of the models involved (e.g., Grainger et al. 2011). On the other hand, the analysis of the percentage of variance explained by each mode in the different ensemble configuration revealed that the modes obtained with the MMEM and the MMEa generally explain larger variances than the observed counterpart while the variance explained by the modes from the SMMEa is generally lower. The latter result implies that the relevance of these modes in the variance associated to the noise is lower than for observations.

The analysis of the sources of predictability revealed that mode activity in DJF is more related to SST anomalies than in JJA, in agreement with previous works (e.g., Mo 2000). In addition, the modes that are more related to tropical SST anomalies are better forecast by the MMEM. The latter could at least partly explain why the main modes of variability are less predictable in JJA than in DJF.

The analysis of the skill and the predictability achieved by the MMEM through these predictable modes reveal that in DJF almost all the MMEM skill can be attributed to the leading modes. However, there are some regions, like South America, in which the MMEM skill is nonsignificant and it could not be improved even if the representation of the predictable modes was perfect. In JJA, the performance of the MMEM could be improved if the representation of the first mode, associated to SAM, was better. Further work needs to be done to explore the reasons of the strong seasonal differences in the SAM skill. It is speculated that the influence that the stratospheric variability in November has in the circulation anomalies in DJF might explain the prediction skill differences between JJA and DJF (e.g., Byrne and Shepherd 2018; Lim et al. 2018). Another explanation for the differences can be the low variance obtained with the MME in polar latitudes during JJA.

The analysis of the 2-m temperature and precipitation anomalies associated with predictable modes showed that the second and third mode are significantly correlated with temperature, mainly in the tropics and midlatitudes, while the third mode is significantly correlated against temperature mostly in the extratropics over continents, Antarctica included. This result could help to improve the forecast made for temperature at surface, especially in extratropical regions where seasonal models are not good at forecasting this variable, like South America (Osman and Vera 2017). On the other 
hand, the correlations between predictable modes and precipitation are significantly mainly over the oceans, especially the Pacific, while over land they are significant only in limited regions.

Some questions remained unanswered and are left to future work, like whether these modes are predictable several months in advance or what the current levels of predictability are in the transition seasons. Future studies using other ensemble data will be done to address these questions.

Acknowledgments. We acknowledge the WCRP/ CLIVAR Working Group on Seasonal to Interannual Prediction (WGSIP) for establishing the Climate-System Historical Forecast Project (CHFP; see Tompkins et al. 2017) and the Centro de Investigaciones del Mar y la Atmosfera (CIMA) for providing the model output (http://chfps.cima.fcen.uba.ar). We also thank the data providers for making the model output available through CHFP. This research was supported by UBACyT 20020170100428BA, PIDDEF 2014/2017 No. 15, Belmont Forum/ANR-15-JCL/-0002-01 "CLIMAX".

\section{REFERENCES}

Arblaster, J. M., and G. A. Meehl, 2006: Contributions of external forcings to southern annular mode trends. J. Climate, 19, 28962905, https://doi.org/10.1175/JCLI3774.1.

Arora, V. K., and Coauthors, 2011: Carbon emission limits required to satisfy future representative concentration pathways of greenhouse gases. Geophys. Res. Lett., 38, L05805, https:// doi.org/10.1029/2010GL046270.

Berman, A. L., G. Silvestri, and R. Compagnucci, 2012: Eastern Patagonia seasonal precipitation: Influence of Southern Hemisphere circulation and links with subtropical South American precipitation. J. Climate, 25, 6781-6795, https:// doi.org/10.1175/JCLI-D-11-00514.1.

Byrne, N. J., and T. G. Shepherd, 2018: Seasonal persistence of circulation anomalies in the Southern Hemisphere stratosphere and its implications for the troposphere. J. Climate, 31, 3467-3483, https://doi.org/10.1175/JCLI-D-17-0557.1.

Colman, R., and Coauthors, 2005: BMRC atmospheric model (BAM) version 3.0: Comparison with mean climatology. BMRC Research Rep. 108, 23 pp.

Fogt, R. L., D. H. Bromwich, and K. M. Hines, 2011: Understanding the SAM influence on the South Pacific ENSO teleconnection. Climate Dyn., 36, 1555-1576, https://doi.org/ 10.1007/s00382-010-0905-0.

Frederiksen, C. S., and X. Zheng, 2007: Variability of seasonalmean fields arising from intraseasonal variability. Part 3: Application to $\mathrm{SH}$ winter and summer circulations. Climate Dyn., 28, 849-866, https://doi.org/10.1007/s00382-006-0214-9.

Ghil, M., and K. Mo, 1991: Intraseasonal oscillations in the global atmosphere. Part II: Southern Hemisphere. J. Atmos. Sci., 48, 780-790, https://doi.org/10.1175/1520-0469(1991)048<0780: IOITGA $>2.0 . \mathrm{CO} ; 2$.

Grainger, S., and Coauthors, 2011: Modes of variability of Southern Hemisphere atmospheric circulation estimated by
AGCMs. Climate Dyn., 36, 473-490, https://doi.org/10.1007/ s00382-009-0720-7.

- C. S. Frederiksen, and X. Zheng, 2013: Modes of interannual variability of Southern Hemisphere atmospheric circulation in CMIP3 models: Assessment and projections. Climate Dyn., 41, 479-500, https://doi.org/10.1007/s00382-012-1659-7.

,$- \ldots$, and — 2014: Assessment of modes of interannual variability of Southern Hemisphere atmospheric circulation in CMIP5 models. J. Climate, 27, 8107-8125, https://doi.org/ 10.1175/JCLI-D-14-00251.1.

Guérémy, J.-F., M. Déqué, A. Braun, and J.-P. Piedelièvre, 2005: Actual and potential skill of seasonal predictions using the CNRM contribution to DEMETER: Coupled versus uncoupled model. Tellus, 57A, 308-319, https://doi.org/10.1111/ j.1600-0870.2005.00101.x.

Hendon, H. H., D. W. J. Thompson, and M. C. Wheeler, 2007: Australian rainfall and surface temperature variations associated with the Southern Hemisphere annular mode. J. Climate, 20, 2452-2467, https://doi.org/10.1175/JCLI4134.1.

Hodson, D. L. R., and R. T. Sutton, 2008: Exploring multi-model atmospheric GCM ensembles with ANOVA. Climate Dyn., 31, 973-986, https://doi.org/10.1007/s00382-008-0372-z.

Jha, B., A. Kumar, and Z.-Z. Hu, 2019: An update on the estimate of predictability of seasonal mean atmospheric variability using North American multi-model ensemble. Climate Dyn., 53, 7397-7409, https://doi.org/10.1007/s00382-016-3217-1.

Kalnay, E., and Coauthors, 1996: The NCEP/NCAR 40-Year Reanalysis Project. Bull. Amer. Meteor. Soc., 77, 437-471, https:// doi.org/10.1175/1520-0477(1996)077<0437:TNYRP>2.0.CO;2.

Kidson, J. W., 1999: Principal modes of Southern Hemisphere lowfrequency variability obtained from NCEP-NCAR reanalyses. J. Climate, 12, 2808-2830, https://doi.org/10.1175/1520-0442(1999) $012<2808$ :PMOSHL $>2.0$. CO; 2 .

Lee, J.-Y., B. Wang, Q. Ding, K.-J. Ha, J.-B. Ahn, A. Kumar, B. Stern, and O. Alves, 2011: How predictable is the Northern Hemisphere summer upper-tropospheric circulation? Climate Dyn., 37, 1189-1203, https://doi.org/10.1007/ s00382-010-0909-9.

_ prediction and predictability of the Asian winter temperature variability. Climate Dyn., 41, 573-587, https://doi.org/10.1007/ s00382-012-1588-5.

Lim, E.-P., H. H. Hendon, and D. W. J. Thompson, 2018: Seasonal evolution of stratosphere-troposphere coupling in the Southern Hemisphere and implications for the predictability of surface climate. J. Geophys. Res., 123, 12 002-12 016, https://doi.org/ 10.1029/2018JD029321.

Marsland, S., H. Haak, J. Jungclaus, M. Latif, and F. Röske, 2003: The Max-Planck-Institute global ocean/sea ice model with orthogonal curvilinear coordinates. Ocean Modell., 5, 91-127, https://doi.org/10.1016/S1463-5003(02)00015-X.

Mo, K. C., 2000: Relationships between low-frequency variability in the Southern Hemisphere and sea surface temperature anomalies. J. Climate, 13, 3599-3610, https://doi.org/10.1175/ 1520-0442(2000)013<3599:RBLFVI > 2.0.CO;2.

Molteni, F., and Coauthors, 2011: The new ECMWF Seasonal Forecast System (system 4). ECMWF Tech. Memo. 656, 49 pp., doi:10.21957/4nery093i.

O'Lenic, E. A., and R. E. Livezey, 1988: Practical considerations in the use of rotated principal component analysis (RPCA) in diagnostic studies of upper-air height fields. Mon. Wea. Rev., 116, 1682-1689, https://doi.org/10.1175/1520-0493(1988)116<1682: PCITUO $>2.0 . \mathrm{CO} ; 2$. 
Osman, M., and C. S. Vera, 2017: Climate predictability and prediction skill on seasonal time scales over South America from CHFP models. Climate Dyn., 49, 2365-2383, https://doi.org/ 10.1007/s00382-016-3444-5.

_ - — , and F. J. Doblas-Reyes, 2016: Predictability of the tropospheric circulation in the Southern Hemisphere from CHFP models. Climate Dyn., 46, 2423-2434, https://doi.org/ 10.1007/s00382-015-2710-2.

Saha, S., and Coauthors, 2006: The NCEP Climate Forecast System. J. Climate, 19, 3483-3517, https://doi.org/10.1175/JCLI3812.1.

Schiller, A., J. S. Godfrey, P. C. McIntosh, G. Meyers, N. R. Smith, O. Alves, G. Wang, and R. Fiedler, 2008: A new version of the Australian community ocean model for seasonal climate prediction. Marine Laboratories Rep. 240, 82 pp., http:// www.cmar.csiro.au/publications/cmrreports/240/abs240.html.

Scinocca, J. F., N. A. McFarlane, M. Lazare, J. Li, and D. Plummer, 2008: Technical note: The CCCma third generation AGCM and its extension into the middle atmosphere. Atmos. Chem. Phys., 8, 7055-7074, https://doi.org/10.5194/acp-87055-2008.

Silvestri, G., and C. Vera, 2009: Nonstationary impacts of the southern annular mode on Southern Hemisphere climate. J. Climate, 22, 6142-6148, https://doi.org/10.1175/2009JCLI3036.1.

Smith, T. M., and R. W. Reynolds, 2004: Improved extended reconstruction of SST (1854-1997). J. Climate, 17, 2466-2477, https:// doi.org/10.1175/1520-0442(2004)017<2466:IEROS>2.0.CO;2.

Stevens, B., and Coauthors, 2013: Atmospheric component of the MPI-M Earth System Model: ECHAM6. J. Adv. Model. Earth Syst., 5, 146-172, https://doi.org/10.1002/jame.20015.

Stockdale, T. N., and Coauthors, 2011: ECMWF Seasonal Forecast System 3 and its prediction of sea surface temperature. Climate Dyn., 37, 455-471, https://doi.org/10.1007/s00382-0100947-3.
Tompkins, A. M., and Coauthors, 2017: The climate-system historical forecast project: Providing open access to seasonal forecast ensembles from centers around the globe. Bull. Amer. Meteor. Soc., 98, 2293-2301, https://doi.org/10.1175/BAMS-D-16-0209.1.

Vera, C., G. Silvestri, V. Barros, and A. Carril, 2004: Differences in El Niño response over the Southern Hemisphere. J. Climate, 17, 1741-1753, https://doi.org/10.1175/1520-0442(2004)017<1741: DIENRO $>2.0 . \mathrm{CO} ; 2$.

von Storch, H., and F. W. Zwiers, 2002: Statistical Analysis in Climate Research. Cambridge University Press, 415 pp., doi:10.1017/CBO9780511612336.

Wang, B., J.-Y. Lee, I. Kang, J. Shukla, S. Hameed, and C. Park, 2007: Coupled predictability of seasonal tropical precipitation. CLIVAR Exchanges, No. 12, International CLIVAR Project Office, Southampton, United Kingdom, 17-18.

Watanabe, M., and Coauthors, 2010: Improved climate simulation by MIROC5: Mean states, variability, and climate sensitivity. J. Climate, 23, 6312-6335, https://doi.org/10.1175/2010JCLI3679.1.

Wu, R., and B. P. Kirtman, 2006: Changes in spread and predictability associated with ENSO in an ensemble coupled GCM. $J$. Climate, 19, 4378-4396, https://doi.org/10.1175/JCLI3872.1.

Xie, P., and P. A. Arkin, 1997: Global precipitation: A 17-year monthly analysis based on gauge observations, satellite estimates, and numerical model outputs. Bull. Amer. Meteor. Soc., 78, 2539-2558, https://doi.org/10.1175/1520-0477(1997) $078<2539$ :GPAYMA $>2.0$. CO;2.

Yukimoto, S., and Coauthors, 2012: A new global climate model of the Meteorological Research Institute: MRI-CGCM3-Model description and basic performance. J. Meteor. Soc. Japan, 90A, 23-64, https://doi.org/10.2151/jmsj.2012-A02.

Zheng, X., and C. S. Frederiksen, 2007: Statistical prediction of seasonal mean Southern Hemisphere 500-hPa geopotential heights. J. Climate, 20, 2791-2809, https://doi.org/10.1175/JCLI4180.1. 